Discussion In this study, the impact of symbols on the understanding of a guidelines recommendation was limited. Important methodological limitations apply but this study questions the impact of symbols to clarify a recommendation's message. However, visual aids are likely to be very important for users to help to identify recommendations within larger text document and therefore make guidelines easier to use and implement.

\section{P093 DETERMINING PHARMACIST AWARENESS AND IMPLEMENTATION OF THE NICE MEDICINES ADHERENCE GUIDELINE}

${ }^{1} \mathrm{~J}$ Thornton, ${ }^{2} \mathrm{~S}$ Tait, ${ }^{2} \mathrm{D}$ Steinke, ${ }^{2} \mathrm{D}$ Ashcroft, N Elliot. ${ }^{1}$ National Institute for Health and Clinical Excellence, Manchester, UK; ${ }^{2}$ School of Pharmacy, University of Manchester, Manchester, UK

\section{0:1136/bmjqs-2013-002293.157}

Background Up to $50 \%$ of medicines are not taken as recommended with potential for reduced benefits or treatment failure and financial implications of the unused medicines. The NICE clinical guideline on Medicines Adherence is relevant to all healthcare workers but there has been no formal assessment of its uptake among pharmacists.

Objectives To determine awareness and application of the Medicines Adherence guideline among UK hospital pharmacists

Method A postal self-completion questionnaire was sent to hospital pharmacies across northwest England. Descriptive statistics were used to analyse the responses and key themes identified from free-text comments.

Results There were 45 responses. Pharmacists were aware of the guideline via communication from NICE (26\%), pharmaceutical/ medical press (20\%) and local communication (14\%). 20\% of respondents reported that their hospital/department had guidance in place before publication of the guideline and 23\% that their hospital issued guidance after publication. 39\% already used the principles of the guideline in their practice and further independent action was not needed whereas $22 \%$ changed their practice. Although most pharmacists considered they had adequate experience and training, insufficient time and technical support were major barriers to addressing adherence issues in practice.

Discussion Improved communication about the guideline is needed. Many pharmacists want to apply the principles of the guideline but need support to overcome barriers to effective implementation.

Implications for Guideline Developers/Users Guideline developers could help implementation by disseminating relevant guideline information more specifically at pharmacists. Cross-referral to the Medicines Adherence guideline could be included in all other relevant NICE guidelines.

\section{P095 THE ROLE OF NATIONAL GUIDELINES IN DEVELOPING REGIONAL WORKING ARRANGEMENTS BETWEEN MEDICAL SPECIALISTS AND GENERAL PRACTITIONERS}

${ }^{1,3} \mathrm{~L}_{\text {Meijer, }}{ }^{1,2} \mathrm{~F}$ Schellevis. ${ }^{1}$ Nivel, Utrecht, Netherlands; ${ }^{2}$ VU University Medical Centre General Practice \& Elderly Care Medicine/EMGO+, Amsterdam, Netherlands; ${ }^{3}$ Medical Coordinating Center Eemland, Amersfoort, Netherlands

10:1136/bmjgs-2013-002293.158

Background In the Dutch health care system, general practitioners (GPs) are gatekeepers for secondary health care. A referral is needed before consulting a medical specialist. In 20 Dutch regions Medical Coordinating centres develop Regional
Agreements (RAs) about patient care at the interface between primary and secondary health care. Ideally, national evidencebased guidelines are used as the basis for RAs.

Objectives To provide insight into the usefulness of national guidelines in the development of RAs.

Methods Qualitative semi-structured interviews were conducted in 2009 with medical coordinators $(\mathrm{N}=9)$, GPs $(\mathrm{N}=16)$ and medical specialists $(\mathrm{N}=14)$, from seven coordinating centres. All participated in developing an RA about different subjects (hematuria, gastroscopy, postmenopausal bleeding, stroke or exercise ECGs). The recorded interviews were transcripted, encoded and analysed in MAXQDA.

Results National guidelines were used in the development of most RAs. GPs and medical specialists reported to use national guidelines from their own (monodisciplinary) organisation. Medical coordinators introduced the most national guidelines. Developing or revising an RA often started on the occasion of a newly published or revised national guideline. The problems in the use of national guidelines are: limited information about cooperation, conflicting information between different guidelines, no trust in the guideline development procedure, and guidelines are not up to date.

Discussion National guidelines have an important role in the development of RAs. National guidelines should pay more attention to recommendations for regional collaboration.

Implications After the development of a national guideline, the developers should keep in touch with GPs and medical specialists in the regions to pick-up their implementation problems.

\section{P096 THE QUALITY OF CLINICAL PRACTICE GUIDELINES OF ACUPUNCTURE}

${ }^{1,2} \mathrm{H}$ Chen, ${ }^{1} Y \mathrm{Gu},{ }^{1} \mathrm{X}$ Zhang, ${ }^{1} \mathrm{G} \mathrm{Li},{ }^{1} \mathrm{~B}$ Xu. ${ }^{1}$ Nanjing University of Chinese Medicine, Nanjing, China; ${ }^{2}$ Chinese GRADE Center, Lanzhou, China

\section{0:1136/bmjqs-2013-002293.159}

Background Acupuncture practice plays an important role in healthcare in China as well as in the world. 2011, the China Academy of the Chinese Medical Sciences (CACMS) published 5 Clinical practice guidelines (CPGS) of acupuncture, which were the only 5 CPGS specialised for acupuncture in China. Our research was to systematically review the quality of these 5 CPGS.

Methods We evaluated the quality of the 5 CPGS through the guideline appraisal instrument: Appraisal of Guidelines for Research and Evaluation II (AGREE II). Four appraisers rated 6 domains separately.

Results None of the included 5(0\%) guidelines described the systematic methods for searching and selecting the evidence, and all $(100 \%)$ appraised the quality of evidence and graded the strength of recommendations. 5 guidelines $(100 \%)$ reported the guideline panel which involved special methodologists, and 5 $(100 \%)$ mentioned updates but no one $(0 \%)$ described a procedure and frequency for updating the guideline. None of the guidelines $(0 \%)$ considered the patients values, and no one $(0 \%)$ used the Grading of Recommendations Assessment, Development and Evaluation (GRADE) system. None (0\%) reported the conflicts of interests. From the assessment with AGREE II, the mean scores were very low for the domains editorial independence $(2 \%)$ and applicability (8\%), while the other domains were low for the rigour of development (18\%), stakeholder involvement (35\%), 'clarity of presentation' (46\%) and 'scope and purpose’ (54\%). 
Implications Now, the quantity of CPGS of acupuncture is very small. As the quality of the guidelines of acupuncture was low, they were still lots of limitations. The research in methodology of the CPGS of acupuncture still must be enhanced.

\section{P097 ADHERENCE TO GUIDELINES FOR PREVENTIVE CHILD HEALTH CARE IN THE NETHERLANDS}

${ }^{1} \mathrm{C}$ Lanting, ${ }^{1} \mathrm{M}$ Fleuren, ${ }^{1} \mathrm{~K}$ Broekhuizen, ${ }^{2} \mathrm{~T}$ Dunnink. 'Netherlands Institute for Applied Scientific Research, Leiden, Netherlands; '2Dutch Centre for Child Health, Utrecht, Netherlands

\section{0:1136/bmjqs-2013-002293.160}

Background Since 1998, several evidence-based guidelines for Preventive Child Health Care (PCHC) were developed at request of the Dutch Ministry of Ministry of Health Welfare and Sports. The Dutch Centre for Child Health oversees the development, implementation and evaluation of guidelines. Adherence to guidelines is a prerequisite for clients to receive the intended care and benefit.

Objective To assess the extent to which professionals in PCHC adhere to key recommendations of nine PCHC-guidelines.

Methods We undertook a survey among a representative sample of PCHC-professionals $(\mathrm{n}=472)$. Guideline developers selected key activities/recommendations in their guideline. Participants indicated, for each key activity, the proportion of children they applied the activity to. From this, we calculated the proportion of all prescribed key activities the professionals had actually adhered to (i.e. completeness of use). Participants marked obstructers to adhere to the guidelines.

Results 299 (63\%) professionals filled-out the questionnaire; 91 doctors, 159 nurses, and 49 medical assistants. Completeness of use varied from $28 \%$ to $98 \%$ per key activity. Lack of time and lack of client cooperation were often mentioned as obstructers.

Discussion There is room for improvement. Problems in measuring adherence have arisen when key recommendations were not very specific.

Implications for Guideline Developers/Users To enable future monitoring, guideline developers should be specific in formulating key activities, and make sure that, in the guideline, key activities are clearly recognisable. Assessment of adherence is recommended as a way to point out weaknesses in guidelines and their implementation.

\section{P098 THE CANADIAN TASK FORCE ON PREVENTIVE HEALTH CARE: PROCESS FOR CRITICAL APPRAISAL OF EXTERNALLY-PRODUCED GUIDELINES}

${ }^{1}$ A Shane, ${ }^{2} \mathrm{M}$ Tonelli, ${ }^{3} \mathrm{R}$ Birtwhistle, ${ }^{4} \mathrm{~K}$ Pottie, ${ }^{2} \mathrm{~N}$ Bell, ${ }^{5,6} \mathrm{H}$ Singh, ${ }^{1} \mathrm{~S}$ Gorber, ${ }^{1} \mathrm{~L}$ Dunfield. ${ }^{1}$ Public Health Agency of Canada, Ottawa, Canada; ' University of Alberta, Edmonton, Canada; ${ }^{3}$ Queen's University, Kingston, Canada; ${ }^{4}$ University of Ottawa, Ottawa, Canada; ${ }^{5}$ University of Manitoba, Winnipeg, Canada; ${ }^{6}$ Cancer Care Manitoba, Winnipeg, Canada

\section{0:1136/bmjqs-2013-002293.161}

Background The Canadian Task Force on Preventive Health Care (CTFPHC) was re-established in 2010, with a mandate to produce clinical practice guidelines (CPGs) for primary preventive care, based on systematic analysis of scientific evidence.

Context In order to increase the range of topics for which the CTFPHC provides guidance, while operating with defined resources, it was necessary to explore alternative dissemination methods. In addition to developing new guidelines and updating existing guidelines, the CTFPHC has developed a process for identifying high quality guidelines produced by other organisations on topics relevant to the CTFPHC's mandate.

Description of Best Practice The process is comprised of 12 steps. When a CPG is identified for appraisal, a literature scan for recently produced guidelines on the same topic by other organisations is conducted. The resultant CPGs are qualitatively assessed based on 5 criteria. The CPG most appropriate for appraisal undergoes AGREEII assessment, followed by development of a summary report that includes a commentary which discusses the strengths, limitations, link between evidence review and CPG and content of the CPG.

Lessons for Guideline Developers, Adaptors, Implementers and/ or Users Through several iterations, a robust process has been developed, with 3 appraisals completed. Two essential steps were identified: systematic selection of guidelines for appraisal, and engagement of KT experts in selecting product format. Because an appraisal identifies CPGs developed using a standard and rigorous methodology, and does not imply the CTFPHC's agreement with the recommendations, it is essential that the summary report contain information required by practitioners to ensure a benefit is gained from its dissemination.

\section{P099 ASSESSMENT OF THE DIFFUSION AND IMPLEMENTATION OF THE CLINICAL PRACTICE GUIDELINES IN MEXICO}

J Sosa-Garcia, S Martinez-Aldana, E Puente-Rosas, D Pineda-Perez, D Hernandez-Santillan. National Center for Health Technology Excellence (CENETEC), Mexico

\section{0:1136/bmjqs-2013-002293.162}

Background The clinical practice guidelines (CPGs) of the National Health System of Mexico (NHSM) aim to improve the quality and to reduce the heterogeneity of medical care.

Objectives To assess the success of the implementation of the CPGs in medical units of the NHSM.

Methods 480 health units were randomly sampled. In each one, the following items were applied: a checklist in order to verify the existence of CPGs, a questionnaire for physicians to document their knowledge, training and awareness on CPGs, and another one to those in charge of the dissemination and implementation process. Clinical records were reviewed to document the use of CPGs.

Results The diffusion of the CPGs is conducted via internet through the Master Catalogue (MC). The MC was identified in $80 \%$ of the sample. The overall level of training on CPGs was $56 \%(45 \%-100 \%) .88 \%$ of doctors are aware of the existence of CPGs and $71 \%$ reported the utilisation of a CPG during the last month. 30\% of the people in charge of dissemination do not have the necessary resources for the dissemination and implementation. The adherence level falls below 50\%.

Discussion One limitation to the dissemination of the CPG is internet access, along with training variability among institutions. The main barrier to the use of CPGs, is the lack of resources (physical material, human and time) for the dissemination and implementation.

Implications for Guideline Developers/Users The knowledge of CPGs is high, however there are barriers that limit their implementation. 